\title{
Factors influencing intrafamilial communication of hereditary breast and ovarian cancer genetic information
}

\author{
Gillian Nycum $^{1}$, Denise Avard ${ }^{*, 1}$ and Bartha M Knoppers ${ }^{1}$ \\ ${ }^{1}$ Centre de recherche en droit public, Université de Montréal, Montreal, Quebec, Canada
}

What factors influence intrafamilial communication of hereditary breast and ovarian cancer (HBOC) genetic risk information? Such information can have health implications for individuals who undergo genetic testing, but it can also have implications for their blood relatives. This literature review adopts an ecological model to summarize factors at the individual, familial, and community levels, as well as cross cutting factors relating to the complexity of $\mathrm{HBOC}$ genetic information and responsibilities that this information can give rise to. These factors are complex and may result in conflicting senses of responsibility. Faced with the task of communicating HBOC genetic information, the response may be to attempt to balance the potential negative impact of the information on the well-being of the informee (eg, can s/he handle this information?) against the potential health benefit that the knowledge could result in. This balancing represents an effort to reconcile conflicting approaches to protecting family members, and is a moral dilemma. This review sheds light on the factors that contribute to resolve this dilemma.

European Journal of Human Genetics (2009) 17, 872-880; doi:10.1038/ejhg.2009.33; published online 25 March 2009

Keywords: BRCA 1/2; ecological; hereditary breast and ovarian cancer; genetic information; family; communication

\section{Introduction}

Hereditary breast and ovarian cancer (HBOC) genetic information has relevance not only for those who undergo genetic testing, but also for their genetic relatives. In light of this, intrafamilial communication of HBOC genetic information is important; but it is also highly complex and many factors weigh into the decision to communicate and the process of communication. This article is a review of factors influencing intrafamilial communication of HBOC genetic information. We adopt an 'ecological' model to

*Correspondence: Denise Avard, Pavillon Maximilien-Caron, 3101, chemin de la tour, Bureau A-9465-9, Montreal, Quebec, H3T 1J7, Canada. Tel: + 1514343 7702; Fax: + 1514343 2122;

E-mail: denise.avard@umontreal.ca

Received 3 June 2008; revised 27 November 2008; accepted 5 February 2009; published online 25 March 2009 categorize influencing factors at the individual, familial and community levels. Cross-cutting factors are also included, such as the complexity of the information and responsibilities that the information can give rise to (see Table 1). Improving the understanding of the factors that influence intrafamilial communication of genetic information may serve to guide genetics professionals and patients in decision making when it comes to intrafamilial communication and assist in the development of policy in this area. It may also help to guide the development of guidelines, particularly as there is a lack of guidance dealing specifically with intrafamilial communication of HBOC genetic information and genetic information more generally. This is especially important given the rapid development of new technologies for generating genetic information and the potential impact of this increase in information on families. 
Table 1 Ecological model: factors influencing intrafamilial communication of HBOC genetic information

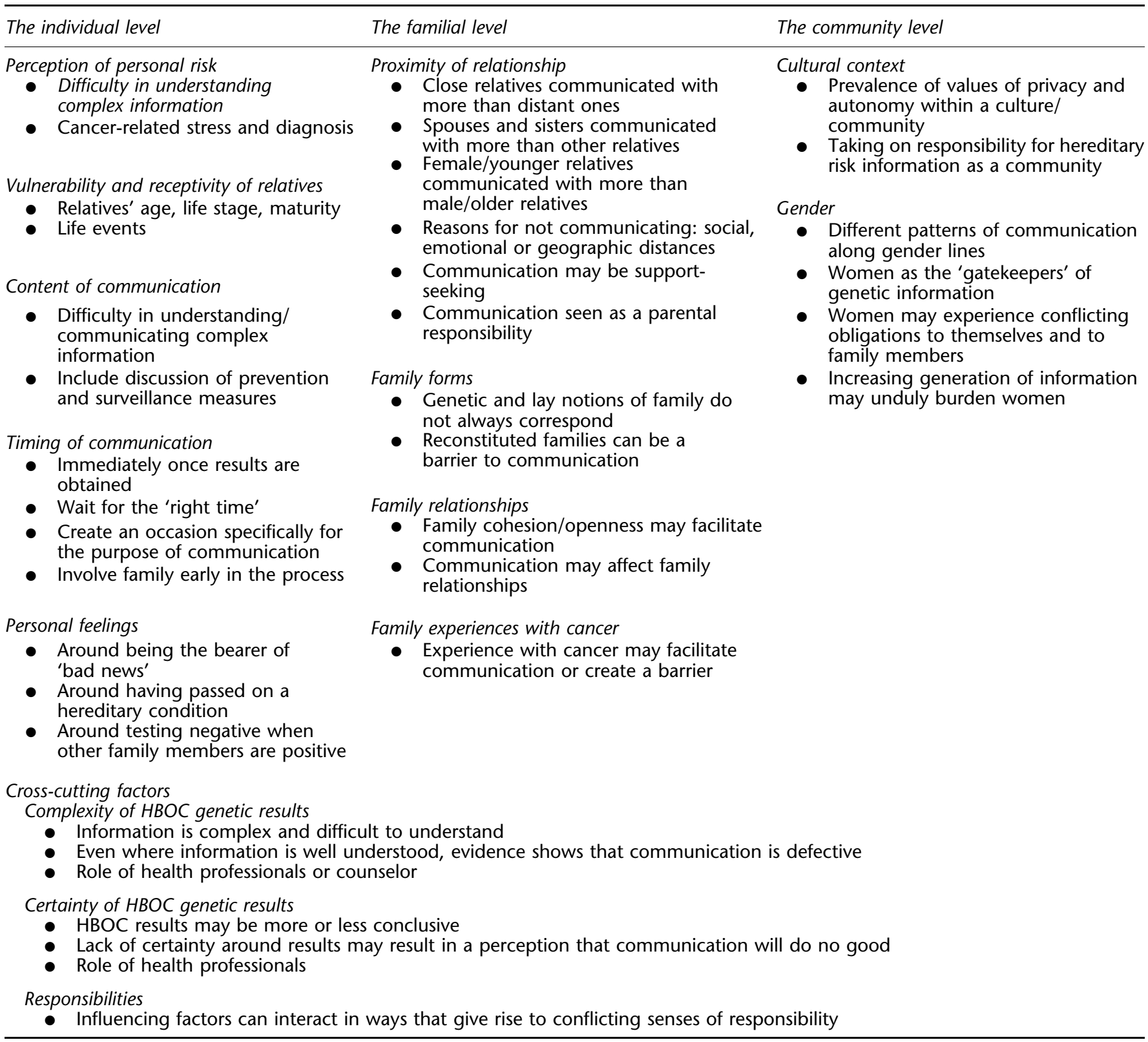

This review is undertaken in the context of HBOC genetic information that living adults obtain in a clinical context. It does not consider communication with minor children, information about deceased adults or information generated in a research context. It also excludes studies of or theories on direct communication with family members by health professionals, ${ }^{1,2}$ considering that this was addressed in another review. ${ }^{3,4}$ Instead, we concentrate on experiences and theories that come strictly out of the familial context.

To inform our review, we relied on qualitative and quantitative research identified in a nonsystematic search for articles published between 1996 and 2008 and indexed by PubMed, article reference lists, Google Scholar, and searches in the gray literature (CDC National Office of Public Health Genomics (http://www.cdc.gov/genomics), the PHG Foundation in Cambridge UK (http://phgfoundation. $\operatorname{org} /$ ) and the HumGen international database of international, regional and national policy (http://www.humgen. umontreal.ca/int/) using the following keywords: BRCA $1 / 2$, HBOC, family, communication and genetic information. Our aim was not to systematically review all existing articles on communication but to describe the factors that 
will help understand how intrafamilial communication transpires.

This article begins by reviewing the factors influencing intrafamilial communication of HBOC information at the individual level; it then considers such factors at the familial and community levels. Finally, it considers cross-cutting factors, such as the complexity of HBOC genetic information, and the responsibilities that this information can give rise to.

\section{The individual level}

An 'individual' in this context is a person who has undergone BRCA1/2 genetic testing for the mutations that indicate a predisposition to HBOC and as such has gained HBOC genetic information that may be of relevance for relatives. In the context of communication of genetic information generally, it has been argued that individuals approach the communication process as a deliberation that may consist of several stages and unfold in several different ways. ${ }^{5,6}$ The deliberative process may include a range of issues such as how to make sense of one's own personal risk, assessing the vulnerability and receptivity of relatives, making decisions about what information should be conveyed, and finally, selecting the right time to communicate the information. ${ }^{6,7}$ Coping with personal feelings, such as guilt and anxiety, may also have an impact on the deliberative process.

\section{Perception of personal risk}

One's own perception of personal risk will impact the weight he or she places on information for self and others, which in turn may impact communication. ${ }^{8}$ As will be discussed in more detail below, reaching an understanding of one's own risk can be difficult because of the uncertainty of some test results; and this can impact the likelihood and accuracy of communication (see section (ii) under Complexity of HBOC genetic information and health professional responsibilities).

Both cancer-related stress and cancer diagnosis may also have an impact on perception of personal risk and communication. One study found that increased cancerrelated stress may lead to difficulty communicating HBOC risk. ${ }^{9}$ In a study involving both women diagnosed with breast cancer as well as unaffected women, affected women underwent genetic testing in an effort to gain a better understanding of the condition and to generate information to help their families, whereas unaffected women tended to want to know their genetic status for their own health. ${ }^{10}$ The authors concluded that cancer diagnosis was a main determinant in the decision to disclose.

\section{Vulnerability and receptivity of relatives}

In determining the vulnerability and receptivity of relatives, considerations about communication are based on the age, maturity level, life stage and perceived risk of the informee. If there is a perception that a relative is not mature enough to understand the information, or is not in an at-risk life stage, communication with them may be withheld. ${ }^{5}$ Some individuals may only inform their children when they have reached an age where preventative measures may be taken or they are at reproductive risk of passing the mutation on. ${ }^{9}$ In a similar vein, if a relative is seen as too old and no longer in a high-risk life stage, there may be no perceived reason to inform them. ${ }^{11}$ Life events, such as graduation or marriage, may also delay communication in an effort to avoid disrupting happy or exacerbating difficult times with potentially alarming information. ${ }^{7}$

\section{Content of communication}

Coming to terms with what should be communicated is another challenge. The particular challenges that arise out of the complexity of genetic information, risk information and genetic concepts will be discussed in more detail below (see section (i) under Complexity of HBOC genetic information and health professional responsibilities). Here, it is worth noting that in a study comparing communication of HBOC genetic information and Huntington's disease (HD) genetic information, it was found that in the case of $\mathrm{HBOC}$, communication was more comprehensive, including discussion of prevention and surveillance measures in some cases; whereas HD discussions were more limited. ${ }^{7}$ Generally, it is well recognized that the comprehensiveness of the communication will differ depending on several factors, such as type of genetic disorder, severity of the disorder, level of predictability and whether treatment or prevention are available.

\section{Timing of communication}

When to communicate genetic information is another step in the deliberation on HBOC communication. HBOC risk was found in one study to be disclosed immediately after the results were obtained. ${ }^{7}$ Some have every intention of informing their relatives, but wait for the 'right time' and feel no sense of urgency around the information; perhaps slipping the information in during an already scheduled family event. ${ }^{6,12,13}$ Others schedule a family meeting where discussion of genetic risk is on the agenda. In some cases, particularly where there is a family history of breast cancer, communicating HBOC genetic information begins before consultation with any genetic services because those who seek BRCA1/2 mutation testing discuss family history with a close family member. ${ }^{14}$ For more accurate HBOC risk assessment, information often needs to be collected from family members before testing, and such conversations facilitate open communication around the issue. ${ }^{6,14-16}$ There is also evidence that involvement of family members in the testing process at an early stage, rather than after results have been returned, may minimizse anxiety associated with the information. ${ }^{17,18}$

\section{Personal feelings}

Some studies report feelings of guilt around disclosing HBOC genetic information. ${ }^{7,19}$ Feelings of guilt may relate 
to being the bearer of perceived 'bad news', ${ }^{20}$ arise with respect to disclosing to a parent who will experience remorse at the knowledge of having passed on a genetic mutation, or be associated with having potentially passed on a genetic condition to offspring. ${ }^{21}$ Guilt associated with passing a mutation to offspring is often accompanied by a faith that biotechnology will find a cure for genetic breast cancer before the disease manifests in children. ${ }^{21}$ Feelings of guilt were also reported when individuals encouraged others to undergo testing and then themselves testing negative, ${ }^{20}$ and in disclosing BRCA $1 / 2$ negative results with family members who had tested positive or who were affected with cancer. ${ }^{20}$

\section{The familial level}

At the familial level, a range of factors influence intrafamilial communication of HBOC risk, including proximity of relationship within the family, lay $v s$ genetic notions of 'family', family relationships and experiences with breast cancer in the family.

\section{Proximity of relationship}

Close relatives, either socially or biologically, are communicated with more often than distant relatives, ${ }^{11,16,22-24}$ and partners and sisters are communicated with more often than other close family members. ${ }^{11,13,24-26}$ Cited reasons for not communicating HBOC genetic information with distant relatives include no, little or superficial contact and/or difficulty in establishing contact, ${ }^{12}$ geographical distance, not knowing the relative personally, and not feeling emotionally close to the relative. ${ }^{12,17,23}$ When more distant relatives are informed, they tend to be female cousins or aunts and younger rather than older. ${ }^{12}$ The same trend is found within the immediate family where communication of BRCA $1 / 2$ test results with female relatives, particularly sisters and daughters, ${ }^{24,27}$ occurs more often than communication with male relatives, such as brothers and sons. ${ }^{6,11,14,26,28}$

One way to account for increased sharing of information with partners, female first-degree relatives and emotionally close relatives is that communicating genetic risk information is to allow support from these family members. Communication as a support-seeking behavior was found in two studies. ${ }^{23,24}$ For example, one study found a slight decrease in psychological distress among those who tested positive for BRCA1/2 and had communicated their genetic risk with a sister, compared with those who did not and experienced a slight increase. ${ }^{14}$ A study of support received from the 'significant others' of women from suspected HBOC families found that although sisters tended to share a close relationship, communication around HBOC could be limited and emotionally strained; male partners were supportive, but on their own terms, or with 'caveats'; and brothers were the most difficult to communicate with. ${ }^{16}$
The study found that friends were the most unconditionally supportive group. $^{16}$ These findings call into question the relationship between communication and support. It may be that communication with partners and sisters is more common because support is 'expected' from them, but that the outcome does not always meet expectations.

Communication of HBOC risk information may be considered a parental responsibility, ${ }^{6,17}$ such that nieces and nephews may not be informed of their risk directly by an aunt or uncle. Rather, this communication will be left to his or her parents; out of respect for intimate family relationships or to avoid the appearance of usurping parental authority. ${ }^{17}$ In cases where a sibling is deceased and his or her family has been reconstituted, there may be a dilemma around ensuring communication with that sibling's children. ${ }^{17}$ In this scenario, there may be a possible contour for perceived intrafamilial responsibility to disclose genetic information. Perhaps disclosure to members of one's own nuclear family exhausts communication responsibilities by transferring them to the sphere of another nuclear family? Once communication with a sibling has occurred, disclosure within the sibling's nuclear family becomes the sibling's own responsibility. These contours both respect the perceived intimate nuclear family sphere and place a limit on perceived responsibility to disclose genetic information to family members. At the same time, it is highly problematic given that 'nuclear' families are uncommon and family constitutions are more complicated than these contours allow.

A lack of social contact with relatives may also result in a sense that there is no, or a lesser, responsibility to communicate with those who may nonetheless be at risk. ${ }^{17,21}$ This may mean that the strength of relationship between relatives may dictate the strength of perceived responsibility that links them. It is important to note however, that the communication barriers discussed here are not barriers for all individuals and families. Many studies reported cases of individuals who went out of their way to disclose to more distant relatives with whom they had no contact or relationship. ${ }^{15,17,29}$

\section{Family forms}

A further layer of complexity follows from the fact that lay or social notions of family and the 'genetic family' do not always correspond. ${ }^{17}$ Rather, there is a tendency to equate familial relation with emotional and social ties. ${ }^{9}$ In one study, siblings were informed of HBOC risk more often than parents and children. ${ }^{11}$ This is interesting because in another study, adult siblings were found to have a harder time understanding HBOC genetic information than adult children. ${ }^{30}$ This may be attributed to the age and perceived vulnerability of parents and children and caution in communicating genetic information with them. ${ }^{14}$ Moreover, the different forms that contemporary families take 
may impact the communication process. Reconstituted families are often at the root of communication barriers. ${ }^{6,15,27,30}$ Family rifts, tensions, divorce, separation and adoption all create barriers to communication of HBOC genetic information. ${ }^{6,15,27,31}$

\section{Family relationships}

Family relationships and concern over the potential impact on family may influence communication. Family cohesion may facilitate communication around BRCA $1 / 2$ results, and more open family communication patterns are likely to extend to HBOC communication and to minimize cancer-related distress. ${ }^{10}$ At the same time, such communication may itself impact or change family relationships, sometimes negatively, by forging a wedge between family members, and sometimes positively by drawing the family closer together. $^{26}$

The potential impact on family relationships is a concern and may affect communication deliberations. ${ }^{31}$ Individuals in one study expressed concern with respect to relatives' reactions that there would be blame, backlash, or a negative impact on relationships. ${ }^{21}$ Some who communicated HBOC genetic risk information reported that they were confronted with anger and blame when coping mechanisms were out of sync. ${ }^{21,22}$ For those who believe that knowledge is power and that genetic information should be shared and acted upon, when relatives respond by employing rationalization and denial as coping mechanisms, the communication could result in anger against informers and cause them distress. ${ }^{21,22}$ When different coping strategies are used, the informed individual may feel coerced to act on the information and undergo testing. ${ }^{22,32}$ Where those who had communicated discovered that family members had felt coerced by them to undergo testing, they reported feeling remorseful. ${ }^{22}$

\section{Familial experiences with cancer}

Experience with breast cancer within a family may also play a role in communication of HBOC risk. ${ }^{9,12}$ The disease may have worked its way into the family script, meaning there is a set way of dealing with it that communication of genetic information may or may not interfere with. ${ }^{33}$ In some families, especially among older generations, the notion of breast cancer as a genetic disease is taboo, and this may interfere with the exchange of family history information across generations. ${ }^{31}$

\section{The community level}

Intrafamilial HBOC communication is sometimes influenced by the cultural context and gender.

\section{Cultural context}

Cultural factors are important in the communication of HBOC genetic information. A few research findings illustrate the influential effect of these cultural factors. At a fundamental level, it is important to note that privacy and individual autonomy are not accorded the same value in all cultural contexts. ${ }^{30}$ The effect may be that personal and genetic information is more readily conceived of as familial information in some cultures. These cultural contexts may facilitate communication of HBOC information.

Apart from privacy issues, one study of the attitudes toward breast cancer genetic testing found that African American women were less concerned about confidentiality protections than Caucasian American women, and were more concerned about access to genetic testing. ${ }^{34}$

As another example, some communities take on responsibility for genetic testing and information dissemination for specific diseases that affect the group. For example, the North American Orthodox Jewish community Dor Yeshorim organization screens members of the community for genetic diseases that commonly afflict its members. Dor Yeshorim uses a coded storage system for the results, and when a prospective marriage match is made, the couple submits to discover their carrier risk status. The results are not communicated to anyone, including testees, unless there is an incompatible match submitted for marriage. ${ }^{35}$ This community approach evades the problem of intrafamilial communication altogether.

\section{Gender}

Gender can also impact communication as women are more often communicated with and more likely to communicate HBOC genetic information with family members. This could be linked in part to the perception that women are at greater risk of developing breast and ovarian cancer than men. ${ }^{17}$ There is generally more anxiety over HBOC risk for female than male descendants. ${ }^{16,18}$ However, men with a BRCA $1 / 2$ mutation are at risk of developing breast cancer (although a lesser risk) and other forms of cancer, ${ }^{33}$ and are at risk of passing the mutation to their offspring. HBOC genetic risk information was found to be more likely to be communicated with a male sibling if he had daughters, and even then the communication tended to be indirect, through a sister-in-law. ${ }^{17}$ These findings support the view that the desire of male members of BRCA $1 / 2$ families to be aware of HBOC risk may be underestimated. ${ }^{18}$ Men with HBOC genetic risk information are more likely to communicate noncarrier status, whereas women communicate carrier and noncarrier status fairly equally; meaning perhaps that men are more comfortable sharing good news than bad. ${ }^{14}$ In HBOC families, there is evidence that men use avoidance as a coping strategy. ${ }^{17}$ There is some evidence that men tested for BRCA $1 / 2$ who are in a relationship with a female partner are more involved in communication of genetic information to their children. ${ }^{18}$ 
Some argue that women are unduly burdened by the intrafamilial implications of genetic information as well as by communication of them. The implications of this burden are that the autonomy of women's decisions to undergo genetic testing and to communicate genetic information may be compromised. Women have been described as the 'gatekeepers of genetic information'; ${ }^{6}$ taking the lead in collecting and exchanging genetic information in the family. Women tend to take responsibility for families, health and care, ${ }^{21}$ including generating and communicating genetic information. ${ }^{17,21}$ Men often rely on women to disclose their genetic test results within their own families more broadly. ${ }^{21}$ Thus, as more genetic information is generated, a disproportionate burden is created for women, ${ }^{21}$ compounded in the context of HBOC cancer genetic information due to perceptions that breast cancer is a woman's disease.

Both Foster et $a^{19}$ and D'Agincourt-Canning ${ }^{20}$ have argued that women experience conflicting senses of responsibility around decisions to undergo genetic testing, and to communicate genetic information. On the one hand, women in that study felt that they have a responsibility to others to provide support, respect relatives' needs and promote relatives' health; but on the other hand, they felt a responsibility to themselves to maintain kin relationships, to take care of their own health needs and to find support. ${ }^{20}$

Similarly, Hallowell et $a l^{34}$ found that women affected with breast cancer undergoing BRCA 1/2 mutation adopted discourses of self-determination and responsibility when accounting for their role in testing and communication. These women saw testing as an opportunity to take up a moral obligation to family members and to act with care. ${ }^{34}$ They also felt that knowledge of genetic risk would provide choice and risk prevention opportunities, and as such understood their role not only as generators and communicators of mutation status information, but also as providers of risk reduction information. ${ }^{34}$

Both studies question whether participants undergo testing and communicate genetic information entirely autonomously, given their feelings of responsibility to communicate and conflicting senses of obligations to themselves and to protect family members from disturbing information. $^{20,34}$ Interestingly, women in the Hallowell study did not see generating information as ethically problematic, but they did recount unforeseen ethical dilemmas with respect to communicating that information with others. Women saw themselves as moral agents, but were not always clear on the right thing to do. In face of dilemmas, participants in the study reconstructed themselves as bearers of bad news, rather than as providers of information that would foster others' autonomy (as they had perceived themselves going in). Moreover, they began to question the value of genetic information and to ask themselves whether in some circumstances, ignorance may be bliss. Having not foreseen the subsequent moral difficulty associated with genetic testing, this study raises the question of whether women's consent to testing is properly informed.

\section{Complexity of HBOC genetic information and health professional responsibilities}

The individual, familial and community factors are intertwined with the physician's communication behavior because health professionals provide social and informational support to families who struggle with the complexity of HBOC genetic test results, with the uncertainty of the results or course of the treatment and with the relevance of the genetic information for the relatives. ${ }^{36}$ Moreover, the generation of HBOC genetic information may give rise to conflicting senses of responsibility.

\section{Complexity of HBOC genetic test results}

HBOC genetic risk information is complex and its implications can be difficult to understand because it relies heavily on the use of statistics and because of the difficulties in understanding the patterns of inheritance of the disease. There is evidence that even when an individual is him or herself well-informed, the transfer of information to relatives is nonetheless highly defective. ${ }^{19,37}$ Moreover, preconceptions about cancer risk may be difficult to shake. In one study, the daughter of a non-BRCA carrier was unequivocal and continued to believe that she was at high risk. ${ }^{31}$ Individuals report feeling that communication of genetic test results is a heavy responsibility, that they feel burdened as the only person available to do it, and worry about their ability to accurately convey the results. ${ }^{14}$ To compound the matter, those on the receiving end seek as much accurate information as possible. ${ }^{19}$ The support of a health professional in communicating with family members may be of assistance. ${ }^{17,38}$ However, there is evidence that health professionals and patients understand and communicate risk in different ways, and guidance for health professionals around how best to communicate genetic risk information may be helpful. ${ }^{39}$

Difficulties understanding patterns of inheritance, as well as family myths about disease inheritance can contribute to communication barriers. ${ }^{9}$ In one study, a general failure to understand the nature of the HBOC genetic information contributed to nondisclosure where an individual at the bottom of the family tree believed that she had no at-risk relatives to pass the information on to, given that she was the youngest. ${ }^{17}$ With complex genetic disorders, such as HBOC, patterns of inheritance may be less intuitively understandable and lead to a lack of clarity as to whom the information is relevant. ${ }^{9}$ Family members will often rely on their health professionals to provide them with the with social, interpersonal, informational and decisional support. 


\section{Certainty of HBOC genetic information}

The certainty or uncertainty associated with hereditary breast cancer risk information can create barriers to communication. Results may be somewhat informative, particularly where results of genetic mutation testing are positive, indicating a higher population risk. However, as HBOC is a complex genetic disease, meaning that genetics, environment and lifestyle factors all play a role, such results do not necessarily provide a clear picture of whether the person will develop breast cancer at some point in their life. In addition, results may be fairly uninformative, such as where there are multiple cases of breast cancer in the family history, but BRCA $1 / 2$ genetic test results are negative. In such cases, a negative test does not rule out hereditary breast or ovarian cancer, but may be associated with an unidentified genetic mutation. Interpreting the implications of this result and attempting to communicate it may be more difficult. HBOC studies have shown that where test results are uninformative (ie, results do not provide clear evidence of the presence or absence of a mutation), the passing on of information to relatives is less likely compared with those who receive more informative results. ${ }^{6,12,24,25,27,30}$ In studies involving women who underwent BRCA $1 / 2$ testing, some women with inconclusive results were hesitant to communicate because they believed that the information would cause increased concern about developing cancer without any potential health benefit. ${ }^{12,25}$ More data are needed on the psychological effect and communication of inconclusive results or results of uncertain significance. ${ }^{40}$

\section{Responsibilities}

Several studies report that individuals experience a sense of responsibility or moral duty to inform family of HBOC genetic risk. ${ }^{12,13,17,20,21,23,24}$ Indeed, one study showed that individuals with suspected HBOC are initially motivated to undergo genetic testing for the express purpose of generating genetic information for relatives. ${ }^{13} \mathrm{~A}$ conflicting obligation reported is the protection of family from psychological or other harm (such as insurance discrimination ${ }^{22,41}$ or causing an upset within the family ${ }^{20}$ ) that may arise from this knowledge. ${ }^{15,18,20,21,31}$ Thus a primary concern around communication may be at the best interests of relatives; however, the complexity of individual perceptions, familial context and relationships, community and cultural context and of genetic information itself may leave individuals unsure how to meet these best interests.

Factors that influence communication of HBOC genetic information are dynamic and interrelated in complex ways; they can overlap and interact in ways that compound and give rise to conflicting senses of responsibility. An example of this was included in the discussion on gender, where a study found that women experienced conflicting senses of responsibility to themselves and to relatives (see section (ii), under The community level). Another example is found in a study where a participant felt unsure whether to communicate her BRCA test results to her daughter who had recently been hospitalized for depression. She felt unsure whether telling her daughter would precipitate a crisis or bring definition to a haunting problem. When she decided not to tell her children, she also felt that she could not inform her adult siblings out of concern that her children would find out through other channels. She worried about the ethics of not disclosing to her adult siblings, and she felt that they had a right to know the information. ${ }^{22}$

\section{Conclusion}

Intrafamilial communication around HBOC genetic risk is a deliberative process with many sources of influence at the individual, familial and community levels, and is influenced by cross-cutting factors. These factors may interact in complex ways, giving rise to conflicting senses of responsibility. In attempting resolution, contextually based and carefully balanced decision making may be undertaken with the goal of acting in accordance with the best interests of at-risk relatives, as well as the family generally. This deliberation may lead to the conclusion that best interests lie in noncommunication. Alternatively, no clear strategy as to how to proceed will emerge, the reaction is passive, and no action is taken to communicate. It is our understanding that noncommunication is rarely an intentional decision, but is rather either passive or a result of careful decision making set deeply within the intrafamilial context.

This literature review has mapped these factors using an 'ecological' model to show how influencing factors interact with one another. The review has also highlighted some areas where more extensive research is required, particularly around the impact of community and cultural context on attitudes toward HBOC genetic information and communication, as well as in the area of communication of inconclusive risk information.

Important elements of this analysis, particularly for those who are grappling with policy development that will touch on intrafamilial communication of HBOC genetic risk information, are (a) the complexity of communication decision making and the multiple factors at play in making any single decision in this context, (b) the need for further understanding of familial responsiveness, social support, and the influence of individual, familial and sociocultural factors and (c) generally, a failure to communicate does not appear to be active or an intentional withholding of information, but rather it is either passive or the outcome of a carefully measured balancing of different approaches to meeting obligations to protect family members and the family environment. Although 
this review included only communication of HBOC genetic information, awareness of the factors that play into communication around this condition and their interconnectedness as shown in the 'ecological' model, will be useful guidance in similar exercises undertaken in the context of other hereditary diseases.

As health professionals typically assume a considerable communication role, their improved understanding of interfamilial communication would theoretically enable them to have a broader understanding of the different issues linked to intrafamilial communication. Nevertheless, health professionals are generally poorly prepared and unaware of the interfamilial communication dynamics. ${ }^{42}$ They need to be in a position to advise their patients. Also the way information is divulged to families' needs careful and sensitive handling, and this is not an easy task. Health professionals are likely to need help and support in considering how to introduce the subject and respond to relatives' questions. ${ }^{43}$ Moreover, the shortage of genetic counselors means that physicians need to be aware of the complex intrafamilial communication dynamics, and think about the families because genetic information affects parents, siblings, children and some time the entire extended family.

\section{Acknowledgements}

This study is supported by the Canadian Breast Cancer Research Alliance.

\section{References}

1 Parker M, Lucassen AM: Genetic information: a joint account? BMJ 2004; 329: 165-167.

2 Suthers GK, Armstrong J, McCormack J, Trott D: Letting the family know: balancing ethics and effectiveness when notifying relatives about genetic testing for a familial disorder. J Med Genet 2006; 43: 665-670.

3 Godard B, Hurlimann T, Letendre M, Egalite N: Guidelines for disclosing genetic information to family members: from development to use. Fam Cancer 2006; 5: 103-116.

4 Lacroix M, Nycum G, Godard B, Knoppers BM: Should physicians warn patients' relatives of genetic risks? CMAJ 2008; 178: $593-595$.

5 Forrest K, Simpson SA, Wilson BJ et al: To tell or not to tell: barriers and facilitators in family communication about genetic risk. Clin Genet 2003; 64: 317-326.

6 Hamilton RJ, Bowers BJ, Williams JK: Disclosing genetic test results to family members. J Nurs Scholarsh 2005; 37: 18-24.

7 Gaff CL, Clarke AJ, Atkinson P et al: Process and outcome in communication of genetic information within families: a systematic review. Eur J Hum Genet 2007)); 15: 999-1011.

8 Wilson BJ, Forrest K, van Teijlingen ER et al: Family communication about genetic risk: the little that is known. Community Genet 2004; $7: 15-24$

9 Van Oostrom I, Meijers-Heijboer H, Duivenvoorden HJ et al: Social and behavioural research in clinical genetics. Clin Genetics 2007; 71: 35-42.

10 Julian-Reynier C, Eisinger F, Chabal F et al: Disclosure to the family of breast/ovarian cancer genetic test results: patient's willingness and associated factors. Am J Med Genet 2000; 94: $13-18$.
11 Claes E, Evers-Kiebooms G, Boogaerts A, Decruyenaere $M$ Denayer L, Legius E: Communication with close and distant relatives in the context of genetic testing for hereditary breast and ovarian cancer in cancer patients. Am J Med Genet A 2003; 116: $11-19$.

12 Van RM, McKinnon WC: Genetic testing for breast and ovarian cancer susceptibility: a family experience. J Midwifery Womens Health 2004; 49: 210-219.

13 Lerman C, Peshkin BN, Hughes C, Isaacs C: Family disclosure in genetic testing for cancer susceptibility: determinants and consequences. J Health Law Policy 1998; 1: 372.

14 Green J, Richards M, Murton F, Statham H, Hallowell N: Family communication and genetic counseling: the case of hereditary breast and ovarian cancer. J Genet Couns 1997; 6: 45-60.

15 Kenen R, rden-Jones A, Eeles R: We are talking, but are they listening? Communication patterns in families with a history of breast/ovarian cancer (HBOC). Psychooncology 2004; 13: 335-345.

16 Keenan KF, Simpson SA, Wilson BJ et al: It's their blood not mine': Whi is responsible for (not) telling relatives about genetic risk? Health Risk Sociology 2005; 7: 209-226.

17 Hallowell N, Ardern-Jones A, Eeles R et al: Communication about genetic testing in families of male BRCA1/2 carriers and noncarriers: patterns, priorities and problems. Clin Genet 2005; 67: 492-502.

18 Sermijn E, Goelen G, Teugels E et al: The impact of proband mediated information dissemination in families with a BRCA1/2 gene mutation. J Med Genet 2004; 41: e23.

19 Foster C, Eeles R, Arden-Jones A, Moynihan C, Watson M: Juggling roles and expectations: dilemmas faced by women talking to relatives about cancer and genetic testing. Psychol Health 2004; 19: 439-455.

20 D'Agincourt-Canning L: Experiences of genetic risk: disclosure and the gendering of responsibility. Bioethics 2001; 15: 231-247.

21 Speice J, McDaniel SH, Rowley PT, Loader S: Family issues in a psychoeducation group for women with a BRCA mutation. Clin Genet 2002; 62: 121-127.

22 McGivern B, Everett J, Yager GG, Baumiller RC, Hafertepen A, Saal HM: Family communication about positive BRCA1 and BRCA2 genetic test results. Genet Med 2004; 6: 503-509.

23 Hughes C, Lerman C, Schwartz M et al: All in the family: evaluation of the process and content of sisters' communication about BRCA1 and BRCA2 genetic test results. Am J Med Genet 2002; 107: $143-150$

24 Patenaude AF, Dorval M, DiGianni LS, Schneider KA, Chittenden A, Garber JE: Sharing BTCA1/2 test results with first degree relatives: Factors predicting whom women tell. J Clin Oncol 2005; 24: 700-706.

25 McKinnon W, Naud S, Ashikaga T, Colletti R, Wood M: Results of an intervention for individuals and families with BRCA mutations: a model for providing medical updates and psychosocial support following genetic testing. J Genet Couns 2007; 16: 433-456.

26 MacDonald DJ, Sarna L, van Servellen G, Bastani R, Giger JN, Weitzel JN: Selection of family members for communication of cancer risk and barriers to this communication before and after genetic cancer risk assessment. Genet Med 2007; 9: 275-282.

27 Smith KR, Zick CD, Mayer RN, Botkin JR: Voluntary disclosure of BRCA1 mutation test results. Genet Test 2002; 6: 89-92.

28 Wagner CJ, Itzen M, Malick J et al: Communication of BRCA1 and BRCA2 results to at-risk relatives: a cancer risk assessment program's experience. Am J Med Genet C Semin Med Genet 2003; 119: $11-18$.

29 Speice J, Harkness J, Laneri $\mathrm{H}$ et al: Involving family members in cancer care: focus group considerations of patients and oncological providers. Psychooncology 2000; 9: 101-112.

30 Adelswad V, Sachs L: The messenger's dilemmas - giving and getting information in genealogical mapping for hereditary cancer. Health Risk Sociol 2003; 5: 125-138.

31 Gilbar R: Communicating genetic information in the family: the familial relationship as the forgotten factor. J Med Ethics 2007; 33: 390-393. 
32 Metcalfe KA, Liede A, Hoodfar E, Scott A, Foulkes WD, Narod SA: An evaluation of needs of female BRCA1 and BRCA2 carriers undergoing genetic counselling. J Med Genet 2000; 37: 866-874.

33 Hallowell N, Foster C, Eeles R, Ardern-Jones A, Murday V, Watson M: Balancing autonomy and responsibility: the ethics of generating and disclosing genetic information. J Med Ethics 2003; 29: $74-79$.

34 Wertz DC: African-Americans' views on ethical issues in genetics: results of a public survey. J Genet Couns 1998; 7: 511-512.

35 Goldrich L: When being incompatible can kill: New Jersey Jewish Standard, New Jersey, 2006 http://www.jstandard.com/index. $\mathrm{php} /$ content/item/2246/\#.

36 Arora NK: Interacting with cancer patients: the significance of physicians' communication behavior. Soc Sci Med 2003; 57: 791-806.

37 Chalmers K, Marles S, Tataryn D, Scott-Findlay S, Serfas K: Reports of information and support needs of daughters and sisters of women with breast cancer. Eur J Cancer Care (Engl) 2003; 12: 81-90.
38 Forrest LE, Delatycki MB, Skene L, Aitken M: Communicating genetic information in families - a review of guidelines and position papers. Eur J Hum Genet 2007; 15: 612-618.

39 O'Doherty K, Suthers GK: Risky communication: pitfalls in counseling about risk, and how to avoid them. J Genet Couns 2007; 16: 409-417.

40 Meiser B: Psychological impact of genetic testing for cancer susceptibility: an update of the literature. Psychooncology 2005; 14: $1060-1074$

41 Lynch HT, Lemon SJ, Durham C et al: A descriptive study of BRCA1 testing and reactions to disclosure of test results. Cancer 1997; 79: 2219-2228.

42 Institute of Medecine: A Model for Delivering Psychosocial Health Services; in Institute of Medecine (ed): Cancer Care for the Whole Patient: Meeting the Psychosocial Needs. Washington DC: The National Academies Press, 2008, pp 153-218.

43 British Medical Association: Human Genetics Choice and Responsibility. Oxford: Oxford University Press, 1998. 